\title{
Geografía del carbono en alta resolución en bosque tropical amazónico del Ecuador utilizando tecnología LiDAR aerotransportada
}

\author{
Xavier Molina S.* \\ Mercedes Farjas A.** \\ Juan Carlos Ojeda M.**
}

Recibido 30 de junio de 2018; aceptado 18 de septiembre de 2018

\begin{abstract}
Estimating biomass of terrestrial vegetation in tropical forest is not only a rapidly expanding research issue, but also a subject of high interest for reducing carbon emissions associated with deforestation and forest degradation (REDD+). The aboveground carbon density estimates (ACD) based on field inventories and airborne sensors, especially LiDAR sensors have led to a substantial progress in large-scale mapping of forest carbon stocks. However, these carbon maps have uncertainties generally associated with the calibration of the regression model used to produce these maps.

This work establishes a methodology for calibrating and validating a general ACD estimation model using LiDAR in Ecuador's Yasuní National Park. The size and location of the plots are considered in the model calibration phase as well as the influence of topography and spatial distribution of biomass. For the adjustment and validation of the model a stratified sampling scheme by topographic positions (valley, slope and ridge) is proposed. The validation of the general model for the study area showed values of $\mathrm{RMSE}=5.81 \mathrm{MgC} \mathrm{ha}^{-1}, \mathrm{R}^{2}=0.94$ and bias $=0.59$, while considering the topographical positions, the model showed values of $\mathrm{RMSE}=1.67 \mathrm{Mg} \mathrm{C} \mathrm{ha}^{-1}$, $\mathrm{R}^{2}=0.98$ and bias $=0.23$ for the valley; $\mathrm{RMSE}=3.13 \mathrm{Mg} \mathrm{C} \mathrm{ha}^{-1}, \mathrm{R}^{2}=0.98$ and

* Instituto Geográfico Militar del Ecuador (IGM), Seniergues E-4 676 y Gral. Telmo Paz y Miño, El Dorado, Quito, Ecuador, correo electrónico: xavier.molina@igm.gob.ec

** Universidad Politécnica de Madrid (UPM), c/Ramiro de Maeztu,7, Madrid 28040, España, correos electrónicos: m.farjas@upm.es; juancarlos.ojeda@upm.es
\end{abstract}


bias $=-0.34$ for the slope; and $\mathrm{RMSE}=2.33 \mathrm{Mg} \mathrm{C} \mathrm{ha}^{-1}, \mathrm{R}^{2}=0.97$ and bias $=0.74$ for the ridge.

The results show that the stratified sampling methodology taking into account topographic positions, effectively calibrates the general model with field estimates of ACD, reducing RMSE and bias. The results show the potential of LiDAR data to characterize the vertical structure of vegetation in a highly diverse forest, allowing accurate estimates of ACD, and knowing continuous spatial patterns of biomass distribution and carbon stocks in the study area.

Key words: forest carbon density, LiDAR, topographic habitats, stratified sampling, tropical forest.

\section{Resumen}

La estimación de la biomasa de la vegetación terrestre en bosque tropical es un tema de gran interés para reducir las emisiones de carbono asociadas a la deforestación y la degradación forestal (REDD+). Las estimaciones de densidad de carbono sobre el suelo (ACD) en base a inventarios de campo y datos provenientes de sensores aerotransportados, en especial con sensores LiDAR, han conducido a un progreso sustancial en el cartografiado a gran escala de las reservas de carbono forestal. Sin embargo, estos mapas de carbono tienen incertidumbres considerables, asociadas generalmente al proceso de calibración del modelo de regresión utilizado para producir los mapas.

En este estudio se establece una metodología para la calibración y validación de un modelo general de estimación de ACD usando LiDAR en el Parque Nacional Yasuní en Ecuador. En el proceso de calibración del modelo se considera el tamaño y la ubicación de las parcelas, la influencia de la topografía y la distribución espacial de la biomasa. Para el ajuste y validación del modelo se propone un esquema de muestreo estratificado por posiciones topográficas (valle, ladera y cima). La validación del modelo general para toda la zona de estudio presentó valores de $\mathrm{RMSE}=5.81 \mathrm{Mg} \mathrm{C} \mathrm{ha}^{-1}, \mathrm{R}^{2}=0.94$ y sesgo $=0.59$, mientras que, al considerar las posiciones topográficas, el modelo presentó valores de $\mathrm{RMSE}=1.67 \mathrm{Mg} \mathrm{C}^{-1}$, $\mathrm{R}^{2}=0.98$ y sesgo $=0.23$ para el valle; $\mathrm{RMSE}=3.13 \mathrm{Mg} \mathrm{C} \mathrm{ha-1}, \mathrm{R}^{2}=0.98 \mathrm{y}$ sesgo $=-0.34$ para la ladera; $\mathrm{xMSE}=2.33 \mathrm{Mg} \mathrm{C} \mathrm{ha}^{-1}, \mathrm{R}^{2}=0.97$ y sesgo $=0.74$ para la cima.

Los resultados muestran el potencial de los datos LiDAR para caracterizar la estructura vertical de la vegetación en un bosque altamente diverso, permitiendo realizar estimaciones precisas de ACD, y conocer patrones espaciales continuos de la distribución de la biomasa aérea y del contenido de carbono en la zona de estudio.

Palabras clave: Densidad de carbono forestal, LiDAR, hábitats topográficos, muestreo estratificado, bosque tropical. 


\section{Resumo}

A estimativa de biomassa da vegetação terrestre em bosque tropical é um tema de grande interesse para reduzir as emissões de carbono associadas ao desmatamento e a degradação florestal (REDD+). As estimativas de densidade de carbono sobre o solo (ACD) com base em inventários de campo e dados provenientes de sensores aerotransportados, em especial com sensores LiDAR, têm conduzido a um progresso substancial na cartografia de grande escala das reservas de carbono florestal. Entretanto, estes mapas de carbono têm incertezas consideráveis, associadas geralmente a processo de calibração do modelo de regressão utilizado para produzir os mapas.

Neste estudo se estabelece uma metodologia para a calibração e validação de um modelo geral de estimação de ACD usando LiDAR no Parque Nacional Yasuní no Equador. No processo de calibração do modelo se considera o tamanho e a localização das parcelas, a influência da topografia e a distribuição espacial da biomassa. Para o ajuste e validação do modelo se propõe um esquema de amostragem estratificada por posições topográficas (vale, encosta e topo). A validação do modelo geral para toda a zona de estudo apresentou valores de $\mathrm{RMSE}=5.81 \mathrm{Mg} \mathrm{C} \mathrm{ha-1,} \mathrm{R}^{2}=0.94$ e viés $=0.59$, enquanto que, ao considerar as posições topográficas, o modelo apresentou valores de $\mathrm{RMSE}=1.67 \mathrm{MgC} \mathrm{ha}{ }^{-1}, \mathrm{R}^{2}=0.98$ e viés=0.23 para o vale; $\mathrm{RMSE}=3.13 \mathrm{Mg} \mathrm{C}^{-1}, \mathrm{R}^{2}=0.98$ e viés $=-0.34$ para a encosta; e $\mathrm{RMSE}=2.33 \mathrm{Mg}$ $\mathrm{C}$ ha $^{-1}, \mathrm{R}^{2}=0.97$ e viés $=0.74$ para o topo.

Os resultados mostram o potencial dos dados LiDAR para caracterizar a estrutura vertical da vegetação em bosque altamente diverso, permitindo realizar estimações precisas de ACD, e conhecer padrões espaciais contínuos da distribuição da biomassa aérea e do conteúdo de carbono na zona de estudo.

Palavras chave: Densidade de carbono florestal, LiDAR, habitats topográficos, amostragem estratificada, bosque tropical.

\section{Introducción}

Los bosques tropicales son importantes reservorios de carbono y biodiversidad, y la caracterización de la distribución espacial de su biomasa aérea (AGB) es un requisito previo para el entendimiento de la dinámica del ciclo de carbono en el tiempo. Se requieren estimaciones exactas de AGB y de los cambios en las existencias de carbono resultado de las actividades humanas para reducir las emisiones derivadas de la deforestación y la degradación forestal (REDD+) para contribuir con los esfuerzos en curso para la mitigación del cambio climático (Angelsen, 2008). En este marco, los bosques tropicales desempeñan un papel vital en el ciclo global del carbono, pero la cantidad de carbono que contienen y su distribución siguen siendo inciertas (Chave et al., 2005). Durante la última década, la estimación de las reservas 
de captación de carbono de los bosques tropicales ha evolucionado a partir de una actividad basada en gran medida en los inventarios de campo (Malhi et al., 2006), hacia un enfoque asistido por teledetección aérea y espacial (Asner et al., 2010).

Las aplicaciones forestales requieren de cartografía de alta precisión que permitan entender las funciones y la dinámica de los bosques, su biodiversidad y el contenido de carbono en la vegetación; especialmente en zonas de extrema fragilidad y alta heterogeneidad como es el caso de los bosques tropicales. Dentro de este campo de estudio, el potencial de LiDAR para identificar y representar el bosque fue reconocido en literatura publicada hace varios años atras, cuando Arp et al., (1982) lo utilizaron para cartografiar bosques en América Central. El Servicio Forestal Canadiense realizó estudios de perfiles con altímetro láser para caracterizar la altura y la cobertura del dosel arbóreo en masas forestales (Aldred y Bonnor, 1985). A principios de la década de los noventa, la NASA empezó a utilizar sistemas de escaneo de onda continua de huella mediana y grande para realizar mediciones verticales de estructura de vegetación en bosques (Dubayah y Drake, 2000). También ha sido utilizado para la caracterización vertical de la estructura forestal (Zimble et al., 2003), la detección de cambios en la vegetación (Yu et al., 2004), la estimación de parámetros forestales para la generación de modelos de propagación de incendios forestales (Erdody y Moskal, 2010), la determinación de modelos del terreno en áreas boscosas (Kraus y Pfeifer, 1998), la extracción de unidades de gestión forestal basados en sensores remotos en bosques tropicales (Hou et al., 2011), y para la caracterización de áreas forestales en regiones tropicales usando OBIA (Dupuy et al., 2013), entre otras aplicaciones.

La tecnología LiDAR ha demostrado ser útil para estimar patrones de densidad de carbono; sin embargo, la precisión y la generalidad de las calibraciones entre las predicciones de densidad de carbono sobre el suelo asistidas por LiDAR y las estimaciones basadas en técnicas de inventario de campo deben incrementarse a fin de hacer el cartografiado de carbono de los bosques tropicales más accesible (Asner y Mascaro, 2014). Los enfoques más comunes para predecir AGB con datos LIDAR se basan en modelos de regresión que vinculan métricas LiDAR a estimaciones de biomasa en parcelas de inventario forestal. El modelo resultante se utiliza entonces para estimar AGB sobre áreas más grandes.

\section{Área de estudio}

La zona geográfica en la que se realiza la investigación, es una parcela de 50 hectáreas localizada en $0^{\circ} 41^{\prime}$ de latitud sur y $76^{\circ} 24^{\prime}$ longitud oeste, al sur del río Tiputini, dentro del Parque Nacional Yasuní en Ecuador (Figura 1). En este parque se concentra una proporción considerable de las especies existentes en bosques tropicales de la Amazonía occidental, convirtiéndolo en uno de los espacios naturales de más alta biodiversidad del mundo (Bass et al., 2010). Debido a esta particularidad y con el fin 
de investigar cómo se mantiene la alta diversidad de árboles de Yasuní y la extrema rareza de sus especies, en 1995 la Universidad Católica del Ecuador, junto con el Instituto Smithsoniano de Estudios Tropicales y la Universidad de Aarhus, seleccionaron una parcela de una extensión de 50 hectáreas en el noroeste del Parque Nacional Yasuní. Esta parcela está asociada a una red mundial de grandes parcelas permanentes CTFS (Center for Tropical Forest Science) con 61 parcelas distribuidas a nivel mundial y dedicadas al estudio de la función de los bosques tropicales y templados y su diversidad ( $</$ www.ctfs.si.edu/ $>$ ).

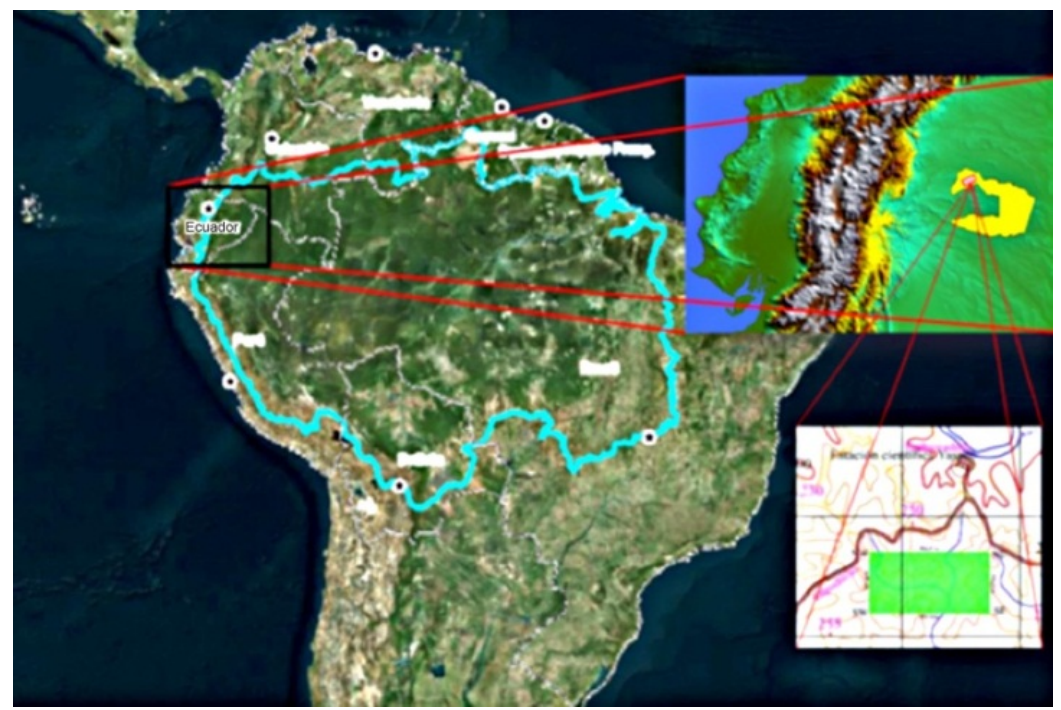

Figura 1. Ubicación de la zona de estudio en la Cuenca Amazónica Occidental. Parcela de 50 ha (rectángulo de color verde) localizada en el Parque Nacional Yasuní, Ecuador (área color amarillo).

La parcela ocupa una superficie de 50 hectáreas y está localizada a una altitud entre los 215 y 249 metros sobre el nivel del mar. La pendiente media de la parcela es del 13\%. Dentro de la parcela se encuentran dos ambientes topográficos característicos y dominantes: el valle y la colina. Las zonas de altitud baja incluyen pequeños arroyos permanentes y pantanos temporales asociados a una depresión (que podrán encontrarse frecuentemente inundadas) localizadas al este de la parcela de estudio. La parcela está delimitada al norte y sur por dos crestas compuestas principalmente por colinas de pendiente suave, aunque con pendientes pronunciadas llenas de cárcavas. Las zonas de baja altitud, contienen aluviones marrones o grises, que separan las crestas entre unas y otras (Valencia et al., 2004). 
Los estudios de suelos dentro de otras zonas del Parque Nacional Yasuní concluyeron que en lugares de elevación alta, la mayoría de suelos son arcillosos, ácidos, ricos en aluminio y hierro, y carentes de rocas y guijarros (Pitman, 2000). La mayoría de los suelos del Parque Nacional Yasuní se clasifican como ultisoles. El resto de los suelos son propensos a inundaciones, ya sea en los pantanos o en las llanuras de inundación, donde predominan los histosoles.

\section{Datos y Modelo general de estimación de carbono}

\section{Censo forestal de la parcela}

El censo de la parcela de estudio fue realizado entre los años 2002 y 2010. La identificación de árboles siguió una metodología estandarizada utilizada en una red mundial de grandes parcelas forestales (Condit, 1998). Durante estos trabajos de campo fueron etiquetadas, identificadas y localizadas todas las plantas leñosas $\geq 1 \mathrm{~cm}$ de diámetro de tallo. Valencia et al. (2004) identificaron todos los árboles con diámetro $\geq 1 \mathrm{~cm}$ en 25 hectáreas de la parcela de estudio. Hasta el año 2010 se realizaron tres censos de individuos de diámetro $\geq 1 \mathrm{~cm}$ en la mitad occidental de la parcela (25 ha) mientras que en el resto de la parcela se realizó un censo de individuos de diámetro $<10 \mathrm{~cm}$ y dos censos de individuos de diámetro $\geq 10 \mathrm{~cm}$. En el censo de 25 hectáreas se identificaron 1104 morfoespecies entre 152353 individuos mientras que en las 50 hectáreas se censaron 341339 individuos.

Se dispuso de mediciones directas del diámetro del tronco a la altura de pecho de todos los individuos de la parcela de 50 hectáreas, y datos de densidad de madera (gravedad específica) tomados de literatura especializada y de mediciones directas en el entorno de la parcela o áreas del PNY (Valencia et al., 2009). En estos censos no se realizaron mediciones de alturas de los árboles debido al dosel denso y cerrado de la zona.

La elevación media del dosel en la parcela es de 18-25 metros. También se encuentran árboles emergentes de hasta $40 \mathrm{~m}$ de altura y en escasas ocasiones árboles de $50 \mathrm{~m}$ de altura. El mayor número de especies de árboles tenían un dosel cuyo tamaño medio tenía una altura de 10 a $20 \mathrm{~m}$, con presencia de árboles de menor tamaño en el sotobosque con alturas máximas de 5-10 m. Las especies de árboles pequeños dominantes en el sotobosque eran de los géneros Matisia y Rinorea, mientras que las especies de dosel importantes fueron las Iriartea deltoidea y Eschweilera coriácea (Valencia et al., 2004b).

\section{Modelo general para estimación de biomasa con variables medidas en campo}

Las estimaciones de biomasa de los bosques tropicales están limitadas por el conocimiento de la alometría de árboles tropicales. Su extrema diversidad de especies de árboles en general impide que se desarrollen alometrías de especies específicas, por lo que generalmente se aplican relaciones generales. La precisión para el cálculo del 
stock de carbono depende de la disponibilidad de modelos alométricos fiables que permitan inferir la biomasa aérea de los árboles en bosque tropical a partir de datos de inventario forestal (Chave et al., 2005, 2014). Por la imposibilidad de desarrollar relaciones alométricas para un área específica de interés, es común utilizar ecuaciones alométricas ya existentes. Debido a la gran cantidad de estudios que documentan tales relaciones (Feldpausch et al., 2012), es importante identificar correctamente las ecuaciones más representativas.

Para la estimación alométrica de la biomasa aérea, se aplicó el enfoque general de Chave et al. (2014). Este modelo (Ecuación 1) se usa en aquellos casos en los que no se cuenta con mediciones de la altura total de los árboles, e incorpora variables como diámetro del tronco, densidad de la madera, y la variable E. Este modelo mejora las predicciones de biomasa propuestos en Chave et al. (2005).

$$
A G B_{\text {est }}=\exp \left[-1.803-0.976 E+0.976 \ln (\rho)+2.673 \ln (D)-0.0299[\ln (D)]^{2}\right]
$$

Donde $D$ representa el diámetro del tronco a la altura de pecho en $\mathrm{cm}, \mathrm{y} \rho$ es la densidad de madera en $\mathrm{g} \mathrm{cm}^{-3}$ de cada árbol. La relación genérica entre el valor de biomasa total sobre el suelo y las características forestales del área de estudio, depende linealmente de una variable de estrés bioclimático $(E)$, que a su vez depende de índices de variabilidad de temperatura, del índice de precipitaciones, y de la intensidad de la sequía.

Con los datos recogidos en el censo y el valor del parámetro $E$ en el Parque Nacional Yasuní (-0.0228121), se calculó la cantidad de biomasa sobre el suelo (en unidades de $\mathrm{Mg} \mathrm{C}$ ha-1), de todos los individuos existentes en la parcela. La sumatoria de las estimaciones en cada árbol, nos dio el valor total de biomasa sobre el suelo para toda la parcela.

\section{Determinación del tamaño de la parcela para el análisis de estimación de carbono}

Es necesario establecer parcelas de gran tamaño para realizar estudios de dinámica del bosque porque abarcan poblaciones importantes de la mayoría de las especies de árboles en la comunidad, especialmente en bosques tropicales (Condit et al., 2000). Hay una tendencia a que los errores en las estimaciones de biomasa disminuyan con el aumento de tamaño de la parcela, ya que parcelas grandes reducen la probabilidad de los efectos de borde, que se producen cuando el dosel de los árboles se encuentra a lo largo de los límites de la parcela. También hay una disminución de errores en general, porque las parcelas grandes capturan una cantidad adecuada de la variabilidad estructural en el campo (Chave y Condit, 2004). Una compilación de investigaciones publicadas sobre estimaciones de biomasa sobre el suelo usando LiDAR (Zolkos et al., 2013) concluye que la incertidumbre se acerca al 10\% cuando el tamaño de la parcela se aproxima a 1 ha. Las estimaciones de biomasa sobre el suelo a escalas espaciales por debajo de 1 ha están dominadas por la incertidumbre, por los 
efectos de corte de parcela y por las diferencias en las características de los sensores (Mascaro et al., 2011). Se admite que escalas espaciales, típicamente del orden de 1 ha, dan resultados más precisos y son más adecuadas en estudios para escalas más finas (Meyer et al., 2013).

Tomando en cuenta los hallazgos de las investigaciones mencionadas, el diseño de muestreo para el análisis fue un cuadriculado regular de subparcelas de 1 ha de superficie cada una de ellas (Figura 2), que permitió capturar el rango de variación de la estructura forestal en la zona de estudio. Las coordenadas X, Y de la esquina SW obtenidas en el levantamiento planimétrico realizado en la parcela de estudio (Figura 3) se usaron como punto de partida; de este modo las subparcelas quedaron georreferenciadas y numeradas de 1 a 50 comenzando en la esquina inferior izquierda.

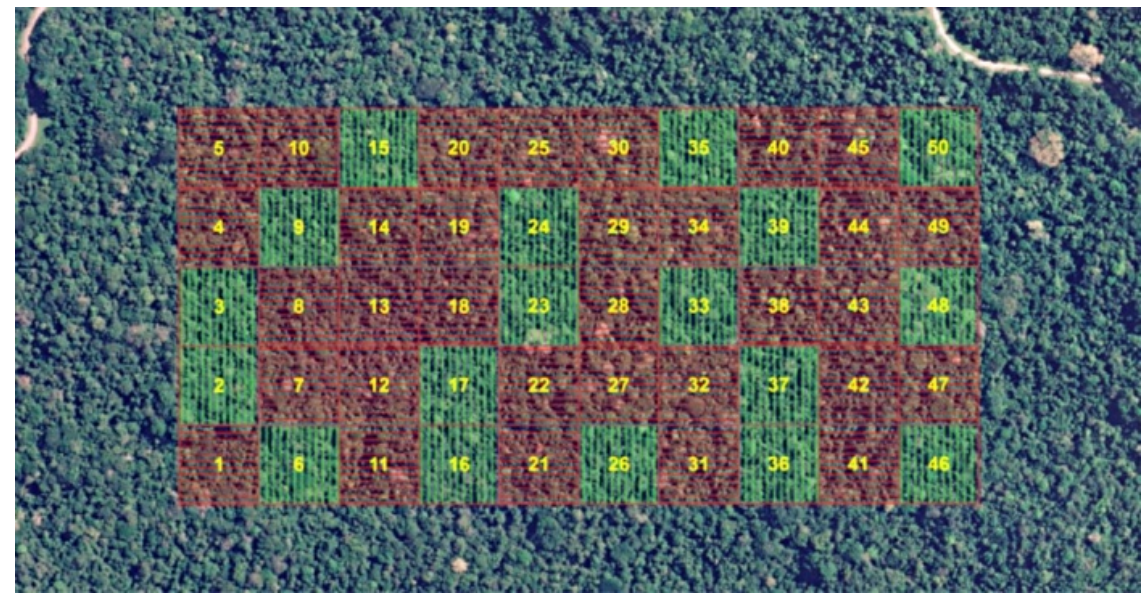

Figura 2. Sistema de cuadriculado regular de subparcelas de 1 ha de superficie, utilizado para el cálculo de la biomasa de la zona de estudio e identificación de las parcelas para el ajuste (trama horizontal roja) y validación (trama vertical verde).

\section{Geoposicionamiento y levantamiento planimétrico de la parcela de estudio}

Para determinar con precisión submétrica los vértices de la parcela de estudio, y relacionar con la máxima exactitud la información del censo con los datos obtenidos mediante el sistema LiDAR aerotransportado, se realizó un levantamiento sobre la parcela de estudio. El Sistema de Referencia Horizontal utilizado en este levantamiento fue el Sistema de Referencia Geocéntrico para las Américas (SIRGAS, Ecuador), compatible con el sistema de posicionamiento por satélite GPS. 
Para el levantamiento planimétrico se implantaron 4 vértices geodésicos (NW-1, NW-2, NE-1, NE-2), que sirvieron de línea base (Figura 3), se utilizó la tecnología GNSS para determinar su ubicación. En la observación se utilizó el vértice geodésico GNSS "Y-NPF”, que forma parte de la Red Nacional GPS del Ecuador (RENAGE). Esta red se encuentra enlazada a SIRGAS (Sistema de Referencia Geocéntrico para las Américas) - ITRF94 (International Terrestrial Reference Frame), época de referencia 1995.4, que como se ha indicado es un marco de referencia compatible con el sistema de posicionamiento satelital GNSS.

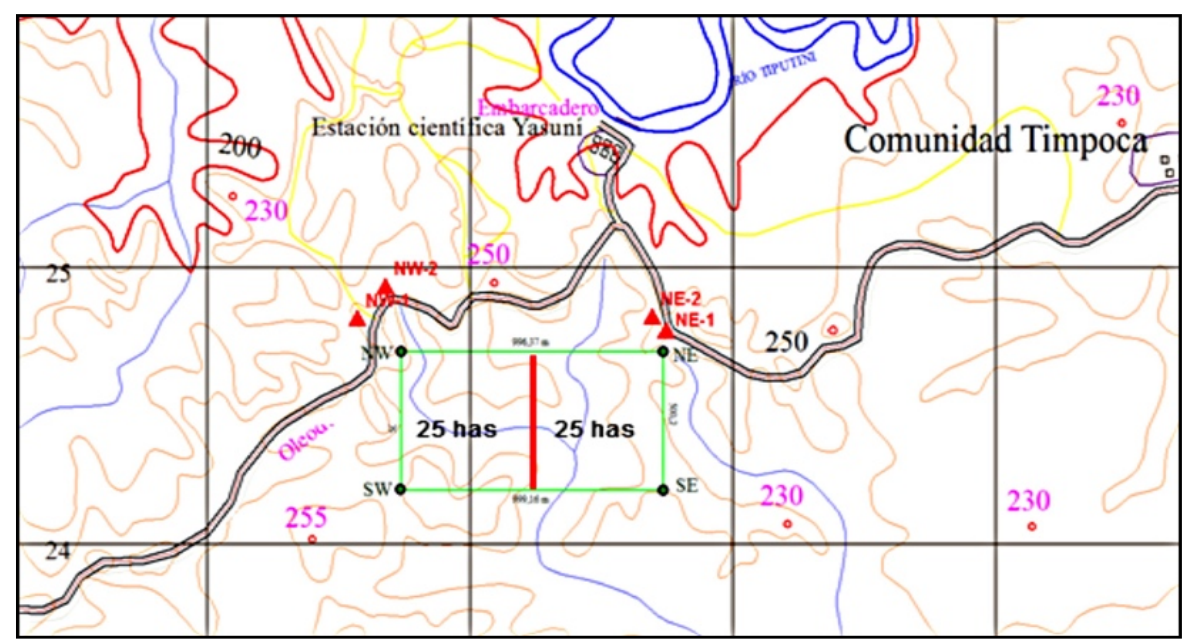

Figura 3. Ubicación de los 4 puntos GNSS y las 4 esquinas de la parcela; Mapa base: C.T Rio Tivacuno, 1:50.000, IGM.

La determinación de las coordenadas de los vértices geodésicos se realizó por el método de posicionamiento GPS estático diferencial de fase, utilizando receptores doble frecuencia marca TRIMBLE, modelo R8, que permiten la medida de líneas base hasta 200 kilómetros, con una precisión de $\pm 0.005 \mathrm{~m}+1 \mathrm{ppm}$. Para el procesamiento de los datos GPS se utilizó el software de procesamiento TRIMBLE BUSINESS CENTER (TBC) Versión 2.5. Para el procesamiento de cada vector GPS, se fijó la precisión horizontal de $<0.050 \mathrm{~m}+1 \mathrm{ppm}$, precisión vertical $<0.100 \mathrm{~m}+$ 1 ppm.

Posteriormente, se realizaron las observaciones necesarias para calcular las coordenadas de las 4 esquinas de la parcela (Tabla 1), mediante una estación total TRIMBLE modelo S3, con una precisión de 2". 
Tabla 1

Coordenadas UTM ZONA 18 sur de las esquinas de la parcela de estudio

\begin{tabular}{ccccc}
\hline Esquinas parcela & Este $(\mathrm{m})$ & Norte $(\mathrm{m})$ & Prec. $(\mathrm{m})$ & Alt. Elip. \\
\hline NW & $343,737.794$ & $9,924,696.411$ & 0.050 & 249.310 \\
NW & $343,735.023$ & $9,924,196.086$ & 0.050 & 252.792 \\
NE & $344,733.085$ & $9,924,695.236$ & 0.050 & 244.899 \\
NE & $344,734.085$ & $9,924,195.180$ & 0.050 & 252.074 \\
\hline
\end{tabular}

Fuente: Elaboración de los autores.

\section{Toma de datos con sensor LiDAR aerotransportado}

Los datos LiDAR fueron colectados en mayo de 2014, la plataforma aérea utilizada fue una avioneta CESSNA 172 modelo Skyhawk que permite cubrir grandes extensiones de terreno a bajas altitudes y velocidades bajas. El equipo LiDAR utilizado fue un Optech ALT Gemini multiretorno. Los datos y configuración del vuelo y equipo se detallan en la Tabla 2.

Tabla 2

Configuración del vuelo y equipo LiDAR

\begin{tabular}{lclc}
\hline \multicolumn{2}{c}{ Datos del vuelo } & \multicolumn{3}{c}{ Configuración LiDAR. } \\
\hline Altitud por encima del suelo $(\mathrm{m})$ & 781.25 & Frecuencia de repet. de pulso (khz) & 66 \\
Distancia entre líneas de vuelo (m) & 203.89 & Frecuencia de escaneo (Hz) & 40 \\
Superposición & $50 \%$ & Angulo de escaneo / FOV & $\pm 15^{\circ}$ \\
Velocidad (m/s) & 56.6 & Densidad nominal de pulsos por $\mathrm{m}^{2}$ & 5.08 \\
Líneas de vuelo & 16 & Ancho de barrido (m) & 407.78 \\
& & Número de retornos & 4 retornos \\
& & Número de ecos o pulso & 2 \\
& & Láser beam divergence (IFOV) & 0.8 \\
& & Espaciado entre puntos (m) & 0.24 \\
& & Densidad de puntos por $\mathrm{m}^{2}$ & 19.4 \\
\hline
\end{tabular}

Fuente: Elaboración de los autores.

En la parcela de 50 hectáreas se registraron 9,603.411 pulsos en total, si bien el número de retornos fueron 4 , el $89.17 \%$ de los datos LiDAR registrados $(8,563.795)$ pertenecieron al primer retorno. Este hecho puede ser explicado por la altura y densidad de la vegetación en la zona de estudio. 


\section{Procesamiento de los datos LiDAR}

La cuantificación de la biomasa sobre el suelo (AGB) depende de las relaciones entre métricas LiDAR y estimaciones de la biomasa a nivel de parcela a partir de mediciones de campo y ecuaciones alométricas (Zolkos et al., 2013). La estimación de la altura del dosel forestal utilizando datos LiDAR depende de una representación exacta de la superficie del suelo en los modelos digitales del terreno (MDT). Para los estudios forestales en particular, la tecnología LiDAR es capaz de caracterizar tanto terreno y la estructura de la vegetación de manera efectiva. Sin embargo, cualquier error en el MDT se propaga y afecta a la precisión de las métricas derivadas de la vegetación (Tinkham et al., 2012) y modelos de altura de dosel.

Los datos LiDAR obtenidos se normalizaron a la superficie del suelo a fin de expresar los retornos en términos de alturas sobre el suelo y se recortaron en forma de malla de 100 m x 100 m usando el software LAStools. A continuación, utilizando el programa FUSION, se calculó un conjunto de datos estadísticos a partir de la nube de puntos LiDAR de cada una de las parcelas de 1 ha y para todo el grupo de parcelas de muestreo (50 has). Estos datos son métricas descriptivas de alturas obtenidas con LiDAR como la mediana, la moda, valores máximos y mínimos, el rango intercuartílico, los distintos percentiles y cuartiles entre otros.

Estas métricas fueron utilizados para relacionar la nube de puntos con la información alométrica del inventario de parcela de campo (diámetro del tronco, área basal, densidad de madera) y para el cálculo de biomasa sobre el suelo calculado con fórmula propuesta por Chave et al. (2014).

\section{Metodología de calibración y ajuste del modelo general}

Las mediciones con LiDAR producen una cobertura detallada de la altura y métricas tridimensionales del dosel que se pueden correlacionar con el área basal, dada la naturaleza volumétrica de las mediciones en 3-D (Drake et al., 2003). La mayoría de los estudios se han centrado hasta el momento en bosques de coníferas y plantaciones forestales en zonas templadas. En esta investigación se analizó esta correlación en los bosques tropicales Amazónicos occidentales que contienen mucha diversidad florística (Bass et al., 2010).

\section{Modelo general de estimación de densidad de carbono sobre el suelo (ACD)}

Al igual que Asner et al. (2012), consideramos que existen tres factores estructurales básicos que describen la cantidad de carbono almacenado en los bosques tropicales: área basimétrica o basal $(\mathrm{AB})$, la altura de los árboles $(\mathrm{H})$ y la densidad específica de la madera (WD). La información obtenida a través de la tecnología LiDAR aerotransportada, está relacionada con las principales variables de masa forestal mediante el ajuste de modelos estadísticos, pudiendo estudiarse variables tales como la altura dominante, el área basimétrica, y la biomasa. 
Para eso es necesario tener dos fuentes de datos: 1) Mediciones dasométricas en parcelas de campo convencionales geoposicionadas con muy alta precisión (submétricas) para garantizar que todas las fuentes de datos disponibles para el estudio coincidan espacialmente; 2) nubes de puntos obtenidos mediante tecnología LiDAR aerotransportada de la zona de estudio, referenciándose de este modo la posición geométrica de la nube de puntos con la información dasométrica de las parcelas. Errores en la identificación y la baja precisión de las variables estimadas, son a menudo atribuidas a discrepancias entre el co-registro de las parcelas de referencia en tierra y la información LiDAR (Næsset, 2002).

\section{Alometría conjunta de parcela}

El enfoque de alometría conjunta de parcela para calibración con LiDAR propuesta por Asner et al. (2012), sigue la teoría general de alometría de árbol (Chave et al., 2005), y refleja las propiedades de la estructura forestal de la parcela completa en conjunto, en lugar de las propiedades de cada organismo en particular. Este enfoque radica en un modelo universal simplificado que demuestra que la biomasa seca de árboles y por lo tanto el contenido de carbono, que es $\sim 48 \%$ de la biomasa seca en peso (Martin y Thomas, 2011), puede estimarse a partir de mediciones de alturas obtenidas con datos LiDAR y otras variables medidas en campo (Asner et al., 2012).

El modelo universal utilizado en este estudio (Ecuación 2) ha sido calibrado y validado experimentalmente en cuatro regiones tropicales: Perú, Panamá, Madagascar y Hawai. El interés de esta investigación es continuar con la validación de este modelo, evaluando si es posible su implementación en otras regiones geográficas o queda relegado a la zona de estudio de la que surgió. El modelo universal se define de la siguiente manera:

$$
A C D=a T C H^{b 1} A B^{b 2} W D_{B A}^{b 3}
$$

Donde la densidad de carbono sobre el suelo $(A C D)$ corresponde al valor de la biomasa sobre el suelo obtenido con la Ecuación 1 y multiplicado por el factor 0.48 . $\mathrm{MCH}$ es la altura media al tope del dosel obtenida con LiDAR, $A B$ es el área basal medida en campo, y $W D_{B A}$ es la densidad de la madera promedio (promedio ponderado con respecto al área basal para cada parcela de 1 ha).

\section{Selección de las subparcelas para el ajuste y validación del modelo general}

Los resultados obtenidos por Réjou-Méchain et al. (2014) refuerzan la importancia de la topografía como un factor que debe ser tomado en cuenta en el diseño de los inventarios forestales y las calibraciones cuando utilizamos sensores remotos. El estudio sugiere que el muestreo en general, debe ser estratificada por hábitats topográficos (por ejemplo, crestas o cimas, valles y laderas), sobre todo si las estimaciones consideran un enfoque basado en el terreno. Por el contrario, cuando la finalidad del 
muestreo de campo es calibrar productos de detección remota, las zonas topográficamente complejas se deben evitar para reducir al mínimo los errores de muestreo asociados a la variabilidad espacial local. En los inventarios forestales y los sistemas de calibración, una importante fuente de error es la distribución desigual y no aleatoria de parcelas en escalas espaciales amplias, especialmente en zonas tropicales.

Para la selección de las subparcelas para el ajuste y validación del modelo en nuestro estudio, se consideró la distribución espacial y hábitats topográficos dentro de la parcela de 50 ha (valle, ladera y cima). Se utilizaron 32 subparcelas para el ajuste y 18 subparcelas para la validación del modelo (Figura 2). La subparcela No. 2 , contiene un remanente de bosque secundario $(0.48 \mathrm{ha})$ resultado de la tala de árboles para la construcción de un antiguo helipuerto abierto en épocas de prospección petrolera en la zona (alrededor del año 1987). Esta subparcela fue considerada en la validación del modelo. En el análisis exploratorio de datos se identificaron dos subparcelas atípicas (la subparcela 36 y la subparcela 50) que contienen los árboles más grandes de la zona. Ambas subparcelas fueron incluidas en la validación del modelo.

Para el ajuste del modelo, se consideraron sólo los árboles $\geq 10 \mathrm{~cm}$ de diámetro a la altura de pecho, ya que los arboles $<10 \mathrm{~cm}$ generalmente contribuyen con menos del 5\% de la biomasa aérea total (AGB) en los bosques tropicales maduros (Chave et $a l ., 2003)$, y es el valor de corte usual en las medidas de inventario forestal y estimaciones de biomasa usando LiDAR (Feldpausch et al., 2011). Para cada una de las subparcelas, se elaboró una tabla con datos del número de árboles, código de especie, diámetro del árbol, ubicación espacial (X,Y), área basal, valor de biomasa estimada (Mg_ha-1) a partir de la fórmula propuesta por Chave et al., (2014), densidad de madera por especie y la densidad de madera ponderado por el área basal (gr_cm-3) para cada uno de los individuos en las subparcelas.

\section{Ajuste del modelo local a partir del modelo general de cálculo de densidad de carbono sobre el suelo (ACD) para la parcela de estudio}

El modelo universal (Ecuación 2) se ajustó mediante regresión lineal múltiple por el método de mínimos cuadrados, transformando logarítmicamente las variables $A C D$, $T C H, A B, y D_{B A}$ en la forma:

$$
\ln (A C D)=\ln a+b_{1} \ln (T C H)+b_{2} \ln (B A)+b_{3} \ln \left(W D_{B A}\right)
$$

Se utilizaron los datos LiDAR y la información dasométrica de 32 subparcelas. El ajuste y diagnosis del modelo se lo realizó con el software $\mathrm{R}$ Commander (Fox, 2005). 


\section{Modelo final de cálculo de ACD}

Luego del análisis y ajuste del modelo general propuesto, el resultado del modelo matemático local que mejor se ajusta es el siguiente:

$$
\ln A C D=\ln (0.768)+0.140 \ln (A B)+0.983\left(W D_{B A}\right)
$$

Los estadísticos obtenidos para cuantificar la bondad de las estimaciones son: el error cuadrático medio (RMSE) igual 0.03182 y el coeficiente de determinación $\mathrm{R}^{2}=0.966$ que determina que porcentaje de la varianza de la variable dependiente es explicado por el modelo de regresión.

A continuación, se revirtió el modelo final ya que nos interesa el parámetro $A C D$ por ha y no su $l n$ y se multiplicó por el factor de corrección (FC) para eliminar el sesgo producido (subestimación) por la transformación logarítmica en el modelo (Baskerville, 1972). El factor de corrección está dado por $\mathrm{CF}=\mathrm{e} \mathrm{MSE} / 2$, donde MSE es el error cuadrático medio del modelo de regresión. Para este caso es igual a 1.00044. La ecuación quedó determinada de la siguiente manera:

$$
A C D=2.15813 * T C H^{0.14015} B A^{1.2292} W D_{P R O M E D I O}^{0.9839}
$$

\section{Validación del modelo local para la estimación de densidad de carbono sobre el suelo(ACD)}

Un procedimiento riguroso de validación del modelo consiste en verificar los resultados con una muestra independiente de la utilizada para construirlo. Para este estudio se realizó la validación del modelo aplicándola a 18 subparcelas que fueron seleccionadas para tal fin. Al aplicar el modelo resultante (Ecuación 5) a las subparcelas de validación, se obtuvo el resultado que se muestra en la Figura 4.

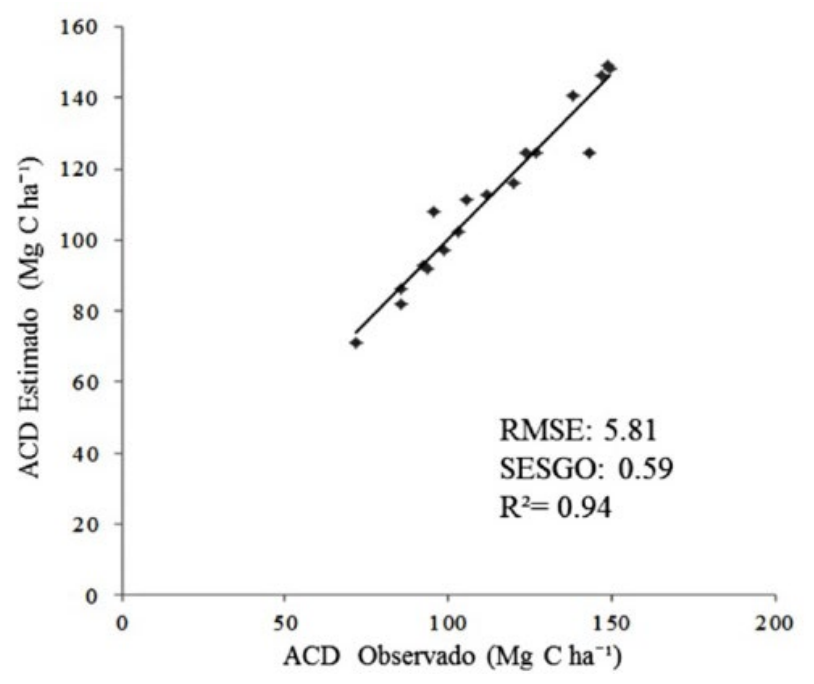

Figura 4. Validación del modelo general de $A C D$ en 18 subparcelas independientes. 
Los resultados de la validación directa ponen de manifiesto que las estimaciones de $A C D$ usando el modelo general son precisas (RMSE $=5.81 \mathrm{Mg} \mathrm{C}$ ha- 1 ), y presentan una alta consistencia con las medidas de campo $(\mathrm{R} 2=0.94)$, sin apenas desviaciones sistemáticas (sesgo próximo a 0 ), recogiendo la variabilidad del ecosistema en la zona de estudio.

\section{Validación del modelo por posiciones topográficas}

En la Figura 5 se presenta el desempeño del modelo ajustado para cada una de las posiciones topográficas. Los resultados muestran que el modelo es espacialmente sensible a las variaciones de $A C D$ en las 3 posiciones topográficas. La selección de subparcelas para el ajuste del modelo considerando estas posiciones, incide en la precisión de las estimaciones de $A C D$ en la zona de estudio. El sesgo en cada posición topográfica proporciona un conocimiento general de cómo las parcelas de campo representan el paisaje. La disminución del sesgo es independiente del número de parcelas de calibración; sólo depende del error de muestreo asociado a estas parcelas, que está determinado en gran medida por el tamaño de la parcela (Réjou-Méchain et al., 2014).
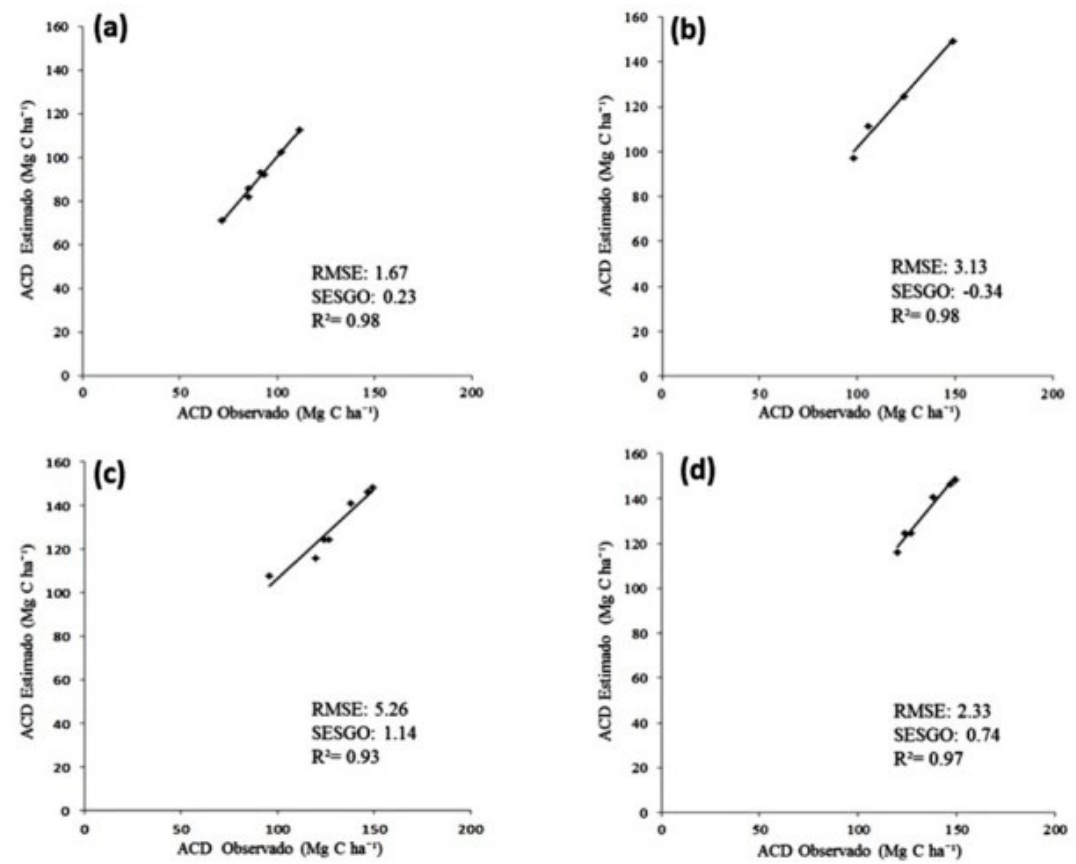

Figura 5. Validación del modelo general de $A C D$ por posiciones topográficas en: a) valle; b) ladera; c) cima incluyendo el remanente de bosque secundario; d) cima sin bosque secundario. 
La mayor diferencia entre las observaciones de campo y las estimadas por el modelo se presenta en la cima que contiene el remanente de bosque secundario (Figura 5c). Esta diferencia se debe a dos situaciones muy concretas: a) el mayor crecimiento de los árboles del bosque secundario en comparación con los árboles del bosque maduro en la parcela; y b) el tiempo transcurridos entre el censo de árboles y la toma de datos LiDAR. La Figura 5d muestra la validación del modelo sin la parcela 2, donde se puede comprobar la mejora en la precisión del modelo.

\section{Geografía del carbono en la zona de estudio}

Los mapas de biomasa y contenido de carbono sobre el suelo de los bosques tropicales son importantes para la conservación de los bosques, ya que constituyen un material básico para la elaboración de políticas de mitigación del cambio climático, y la comprensión del papel que desempeñan los bosques tropicales en el ciclo global del carbono.

Para obtener el mapa de alta resolución de las reservas de carbono en el área de estudio, se aplicó la Ecuación 5 a toda la superficie de cobertura LiDAR utilizando algebra de mapas. La zona total de cobertura LiDAR se dividió en unidades de análisis de 1 ha (malla regular de celdas cuadradas -fishnet), y se calculó el TCH (Figura 6) a partir del modelo de digital de superficie normalizado.

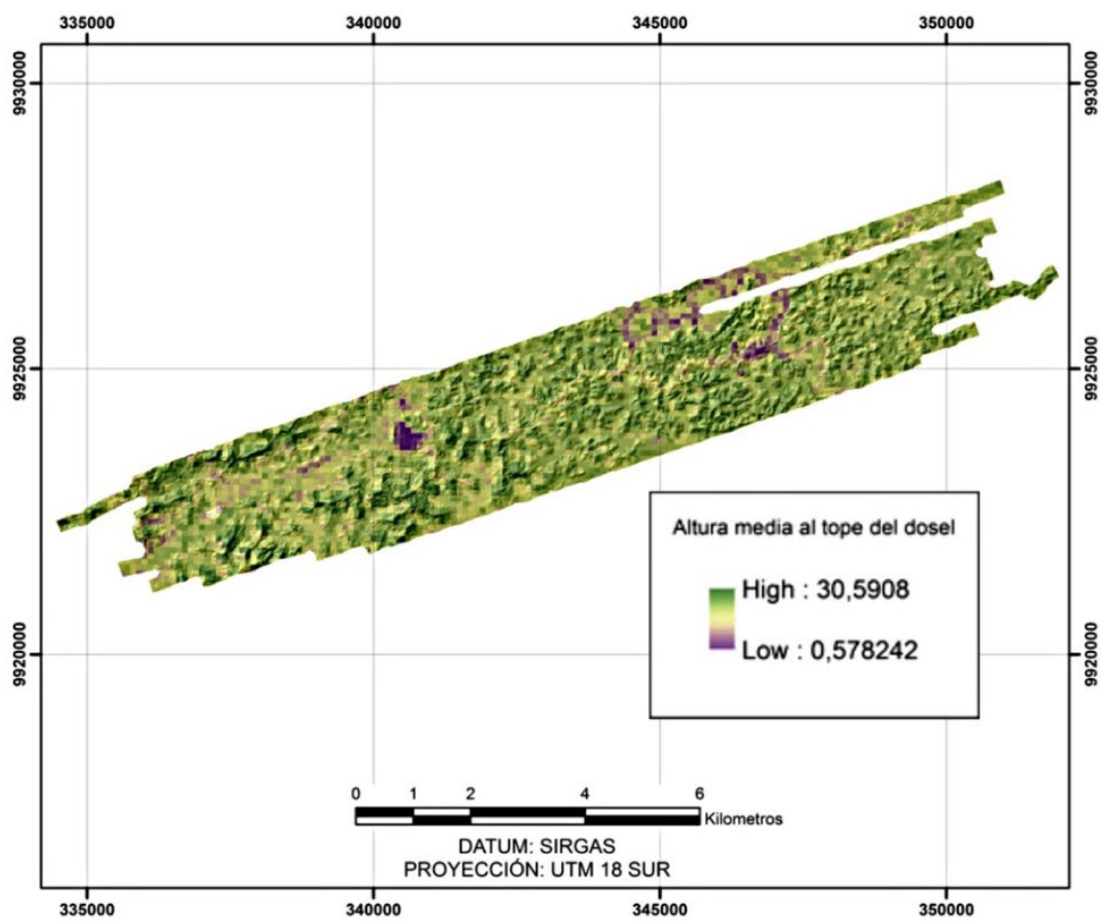

Figura 6. Mapa de altura media al tope del dosel $(\mathrm{TCH})$ para la zona de estudio 
El mapa resultante de la densidad de carbono sobre el suelo (Figura 7), a pesar de cubrir una pequeña extensión de territorio, es una muestra representativa de la diversidad forestal del Parque Nacional Yasuní, y consecuentemente de una gran parte del bosque tropical amazónico del Ecuador.

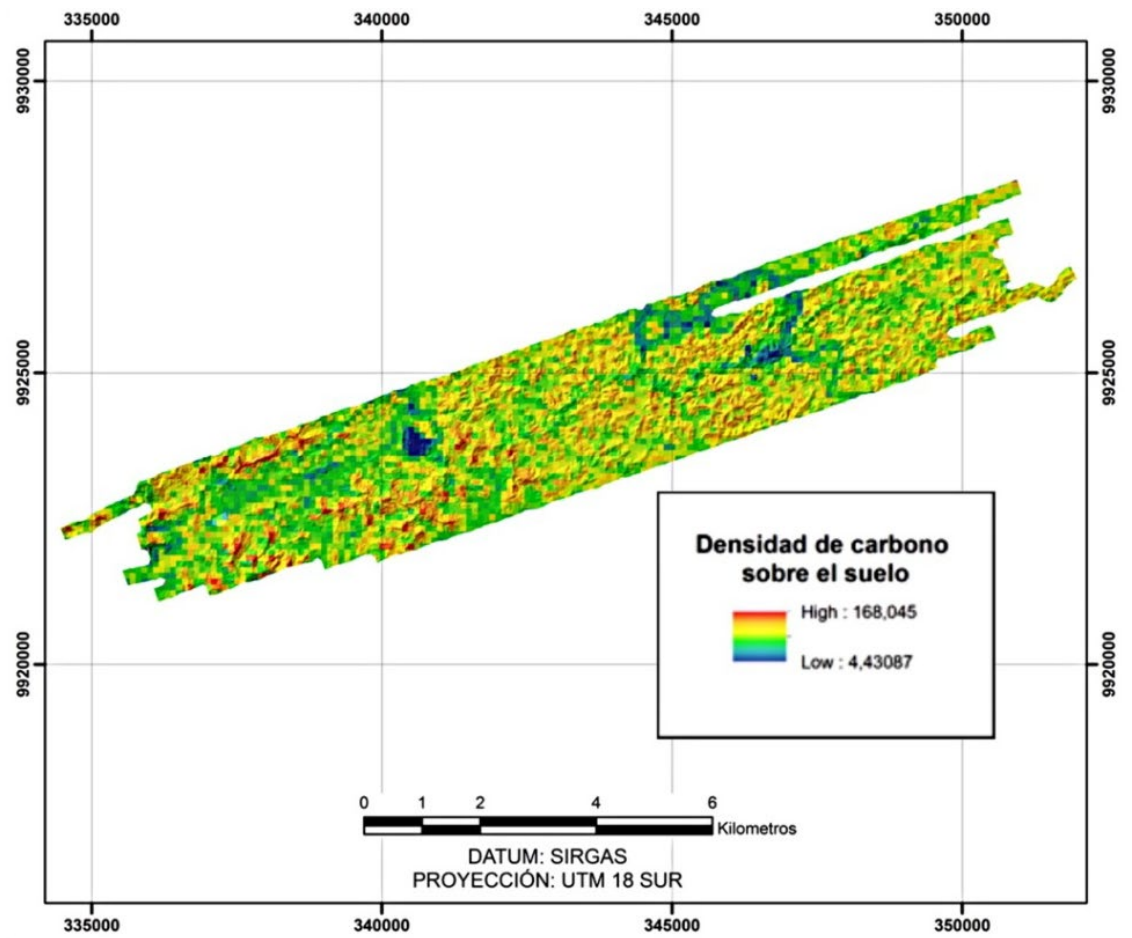

Figura 7. Mapa de densidad de carbono sobre el suelo en la zona de estudio.

Los valores más altos de ACD (168 Mg C ha-1) se localizan en los bosques ubicados en los sitios más elevados (cimas), mientras que las menores densidades de $\mathrm{ACD}$ se encuentran en los bosques en los valles ( $82 \mathrm{Mg} \mathrm{C}$ ha-1), y en zonas inundadas, o propensas a inundaciones (47 Mg C ha-1). Las zonas con almacenamiento bajo de carbono se deben probablemente a las condiciones hidrológicas como la anoxia, y posiblemente a cambios en la fertilidad del suelo asociados con el substrato geológico subyacente (Asner et al., 2010). Los resultados obtenidos concuerdan con el estudio realizado por el Ministerio del Ambiente de Perú el año 2014 en la Amazonía Norte del Perú, donde encontraron que las mayores densidades de carbono se localizan al norte del río Napo, en el límite fronterizo con el Ecuador. 


\section{Conclusiones}

El uso de información proveniente de nuevas tecnologías de captura de datos como el LiDAR aerotransportado, unido a la información de las características biofísicas de los árboles obtenidas en parcelas de campo tácticamente ubicadas y representativas de la diversidad biológica, combinadas con técnicas de modelaje geo-estadístico, han demostrado ser un enfoque consistente para la calibración de datos LiDAR aplicados al estudio del carbono contenido en la biomasa de los bosques tropicales. A través de esta investigación, se comprobó que los sistemas LiDAR aerotransportados son capaces de capturar la variabilidad de la estructura vertical de un bosque tropical de gran biodiversidad en la Amazonía occidental del Ecuador.

La incertidumbre de los resultados obtenidos en estudios realizados hasta el momento, varían desde 17 hasta más de $40 \mathrm{Mg} \mathrm{C}$ ha-1 (RMSE) en los trópicos. Estos errores están relacionados con el proceso de calibración (Asner et al., 2010); en esta investigación se lograron reducir estos valores usando técnicas de regresión lineal combinadas con una metodología para determinar la ubicación más adecuada de las parcelas para el ajuste considerando los hábitats topográficos de la zona de estudio.

Para el cálculo de la densidad de carbono sobre el suelo se utilizaron 50 subparcelas de 1 ha de extensión cada una, obteniendo valores de incertidumbre de $5.81 \mathrm{Mg}$ $\mathrm{C}$ ha-1 que mejoran los resultados de estudios anteriores y ratifican que usando LiDAR se pueden cartografiar existencias de carbono con $\sim 10 \%$ de error y con alta precisión.

El modelo estimado utilizó la densidad de madera promedio por parcela en vez de la densidad de madera ponderado por área basal que utiliza Asner et al. (2012), ya que permitió un mejor ajuste como se puede evidenciar en los resultados de las estadísticas de bondad de ajuste.

El modelo estimado en este estudio recoge la variabilidad de la biomasa sobre el suelo de los tres hábitats topográficos de la zona en conjunto: valle, ladera y colina. Se comprobó que existen diferencias hasta del 50\% en los valores de biomasa aérea entre hábitats en una variación altitudinal de tan solo 32 metros. Los resultados obtenidos confirman las estimaciones realizadas por Valencia et al. (2009). Esta variación de biomasa parece no sólo depender de factores biológicos como la altura, el área basimétrica y la densidad de madera, sino también de la influencia del gradiente de elevación que produciría acumulación de agua en el suelo y la formación de pantanos, que coincide con aquellas zonas de baja pendiente y altitud. Se deben considerar análisis más detallados sobre la influencia de la topografía en las estimaciones de biomasa aérea y que tomen en cuenta un esquema de gradiente, utilizando productos de alta precisión derivados de las mediciones de los sistemas LiDAR en futuras investigaciones.

En el área de estudio y durante el proceso de validación del modelo se identificó subparcelas con muy pocos árboles de dosel emergente dispersos que alcanzaron los 
$56 \mathrm{~m}$ de altura; es de destacar que estos tipos de árboles contienen mucha biomasa aérea y pueden ser fácilmente identificados utilizando la información LiDAR.

\section{Bibliografía}

Aldred, A. y Bonnor, G. (1985). Application of airborne lasers to forest surveys, Petawawa National Forestry Institute, vol. PI-X.51.

Angelsen, A. (2008). Moving ahead with REDD: issues, options and implications, Bogor, Indonesia, Center for International Forestry Research (CIFOR).

Arp, H. y Tranarg, C. (1982). "Mapping in tropical forests: a new approach using the laser APR [Airborne Profile Recorder], Photogrammetric Engineering and Remote Sensing, no. 48.

Asner, G. y Mascaro, J. (2014). "Mapping tropical forest carbon: Calibrating plot estimates to a simple LiDAR metric", Remote Sensing of Environment, no. 140, pp. 614-624.

Asner, G.; Mascaro, J.; Muller-Landau, C.; Vieilledent, G.; Vaudry, R.; Rasamoelina, M., et al. (2011). "A universal airborne LiDAR approach for tropical forest carbon mapping”, Oecologia, 168(4), pp. 1147-1160 $<\mathrm{http}$ :/dx.doi.org/10.1007/s00442-011-2165-z>.

Asner, G.; Powell, N.; Mascaro, J.; Knapp, D.; Clark, K.; Jacobson, J. et al. (2010). "High-resolution forest carbon stocks and emissions in the Amazon", Proceedings of the National Academy of Sciences of the United States of America, 107 (38), pp. 16738-42.

Baskerville, G. (1972). "Use of logarithmic Regression in the Estimation of Plant Biomass", Canadian Journal of Forest Research, no. 2, pp. 49-53.

Bass, S.; Finer, M.; Jenkins, N.; Kreft, H.; Cisneros-Heredia, F.; McCracken, F.; et al. (2010). "Global conservation significance of Ecuador's Yasuní National Park", PloS One, 5(1), pp. 1-22.

Chave, J.; Andalo, C.; Brown, S.; Cairns, A.; Chambers, Q.; Eamus, D. et al. (2005). "Tree allometry and improved estimation of carbon stocks and balance in tropical forests", Oecologia, 145 (1), pp. 87-99.

Chave, J.; Condit, S.; Aguilar, A.; Hernandez, S.; Lao, and R. Perez (2004). "Error propagation and scaling for tropical forest biomass estimates", Philosophical Transactions of the Royal Society B: Biological Sciences, no 359, pp. 409-420.

Chave, J.; Condit, R.; Lao, S.; Caspersen, P.; Foster, B. and Hubbell, S. (2003). "Spatial and temporal variation of biomass in a tropical $\backslash$ rforest: results from a large census plot in Panama", Journal of Ecology, 91(2), pp. 240-252.

Chave, J.; Réjou-Méchain, M.; Búrquez, A.; Chidumayo, E.; Colgan, M.S.; Delitti, C. et al. (2014). "Improved allometric models to estimate the aboveground biomass of tropical trees", Global Change Biology, pp. 1-14.

Condit, R. (1998). Tropical forest census plots. Berlin, Springer-Verlag. 
Condit, R.; Ashton, S.; Baker, P.; Bunyavejchewin, S.; Gunatilleke, S.; Gunatilleke, N. et al. (2000). "Spatial patterns in the distribution of tropical tree species", Science, 288(5470), pp. 1414-1418.

Drake, J.; Knox, G.; Dubayah, R.; Clark, B.; Condit, R.; Blair, B. and Hofton, M. (2003). "Above-ground biomass estimation in closed canopy Neotropical forests using lidar remote sensing: factors affecting the generality of relationships", Global Ecology and Biogeography, 12(2), pp. 147-159.

Dubayah, R. y Drake, J. (2000). "Lidar remote sensing for forestry", Journal of Forestry, 98(6), pp. 44-46.

Dupuy, S.; Lainé, G.; Tassin, J.; y Sarrailh, J. (2013). "Characterization of the horizontal structure of the tropical forest canopy using object-based LiDAR and multispectral image analysis". International Journal of Applied Earth Observation and Geoinformation, no. 25, pp. 76-86.

Erdody, L., y Moskal, M. (2010). "Fusion of LiDAR and imagery for estimating forest canopy fuels", Remote Sensing of Environment, 114(4), pp. 725-737.

Feldpausch, R.; Lloyd, J.; Lewis, L.; Brienen, W.; Gloor, M.; Monteagudo-Mendoza, A. et al. (2012). "Tree height integrated into pantropical forest biomass estimates", Biogeosciences, 9(8), pp. 3381-3403.

Fox, J. (2005). "The R Commander: A basic-Statistics Graphical User interface to R", Journal of Statistical Software, 14 (9), pp. 1-42.

Hou, Z.; Xu, Q. y Tokola, T. (2011). "Use of ALS, Airborne OR and ALOS AVNIR2 data for estimating tropical forest attributes in Lao PDR", Isprs Journal of Photogrammetry and Remote Sensing, 66(6), pp. 776-786.

Kraus, K., y Pfeifer, N. (1998). "Determination of terrain models in wooded areas with airborne laser scanner data", ISPRS Journal of Photogrammetry and Remote Sensing, 53(4), pp. 193-203.

Malhi, Y.; Wood, D.; Baker, T.R.; Wright, J.; Phillips, O.L.; Cochrane, T. et al. (2006). "The regional variation of aboveground live biomass in old-growth Amazonian forests", Global Change Biology, 12(7), pp. 1107-1138.

Martin, R. y Thomas, C. (2011). "A reassessment of carbon content in tropical trees", PloS One, 6(8), e23533.

Mascaro, J.; Detto, M.; Asner, G. and Muller-Landau, H. (2011). "Evaluating uncertainty in mapping forest carbon with airborne LiDAR". Remote Sensing of Environment, 115 (12), pp. 3770-3774.

Meyer, V.; Saatchi, S.; Chave, J.; Dalling, J.; Bohlman, S.; Fricker, A.; Robinson, C.; Neumann, M. y Hubbell, S. (2013). "Detecting tropical forest biomass dynamics from repeated airborne lidar measurements", Biogeosciences, 10, pp. 5421-5438.

Næsset, E. (2002). "Predicting forest stand characteristics with airborne scanning laser using a practical two-stage procedure and field data", Remote Sensing of Environment, 80(1), pp 88-99. 
Pitman, N. (2000). A large-scale inventory of two Amazonian tree communities, Duke University.

Réjou-Méchain, M.; Muller-Landau, C.; Detto, M.; Thomas, C.; Le Toan, T.; Saatchi, S. et al. (2014). "Local spatial structure of forest biomass and its consequences for remote sensing of carbon stocks", Biogeosciences Discussions, 11, pp. 57115742.

Tinkham, T.; Smith, S.; Hoffman, C.; Hudak, T.; Falkowski, J.; Swanson, E. and Gessler, P. (2012). "Investigating the influence of LiDAR ground surface errors on the utility of derived forest inventories", Canadian Journal of Forest Research, 42, pp. 413-422.

Valencia, R.; Condit, R.; Foster, B.; Romoleroux, K.; Villa-Munoz, G.; Svenning, J. et al. (2004). "Yasuní Forest Dynamics Plot, Ecuador", in E.C. Losos and E.G. Leigh (eds.), "Tropical forest diversity and dynamism: Findings from a largescale plot network", Chicago, University of Chicago Press, pp. 609-628).

Valencia, R.; Condit, R.; Muller-Landau, H.; Hernandez, C. y Navarrete, H. (2009). "Dissecting biomass dynamics in a large Amazonian forest plot", Journal of Tropical Ecology, 25(5), pp. 473-482.

Valencia, R.; Foster, B.; Villa, G.; Condit, R.; Svenning, J.; Hernández, C. et al. (2004b). "Tree species distributions and local habitat variation in the Amazon: large forest plot in eastern Ecuador", Journal of Ecology, 92(2), pp. 214-229.

Yu, W.; Hyyppa, J.; Kaartinen, H. y Maltamo, M. (2004). "Automatic detection of harvested trees and determination of forest growth using airborne laser scanning", Remote Sensing of Environment, 90(4), pp. 451-462.

Zimble, A.; Evans, L.; Carlson, C.; Parker, C.; Grado, C. and Gerard, D. (2003). "Characterizing vertical forest structure using small-footprint airborne LiDAR", Remote Sensing of Environment, 87(2), pp. 171-182.

Zolkos, S.; Goetz, S. and Dubayah, R. (2013). "A meta-analysis of terrestrial aboveground biomass estimation using lidar remote sensing", Remote Sensing of Environment, no. 128 , pp. 289-298. 